\title{
ON ALMOST STABLE CMC HYPERSURFACES IN MANIFOLDS OF BOUNDED SECTIONAL CURVATURE
}

\author{
JULIEN ROTH AND ABHITOSH UPADHYAY
}

\begin{abstract}
We show that almost stable constant mean curvature hypersurfaces contained in a sufficiently small ball of a manifold of bounded sectional curvature are close to geodesic spheres.
\end{abstract}

\section{Introduction AND StAtement of the Result}

Let $\phi:\left(M^{n}, g\right) \rightarrow\left(N^{n+1}, \bar{g}\right)$ be an isometric immersion of an oriented closed $n$ dimensional Riemannian manifold $M$ in a $(n+1)$-dimensional oriented manifold $(N, \bar{g})$. We assume that $M$ is oriented by the global unit normal field $\nu$ so that $\nu$ is compatible with the orientations of $M$ and $N$. We will denote by $B$ the second fundamental form of $\phi$ and its mean curvature by $H$. Let $F:(-\varepsilon, \varepsilon) \times M \longrightarrow N$ be a variation of $\phi$ so that $F(0,)=.\phi$. The balance volume associated with the variation $F$ is the function $V:(-\varepsilon, \varepsilon) \longrightarrow \mathbb{R}$ defined by

$$
\int_{[0, t] \times M} F^{\star} d v_{\bar{g}}
$$

where $d v_{\bar{g}}$ is the volume element associated to the metric $\bar{g}$ on $N$. We will denote simply by $d v$ the volume element of $g$. It is a classical fact that

$$
V^{\prime}(0)=\int_{M} f d v
$$

where $f(x)=\left\langle\frac{\partial F}{\partial t}(0, x), \nu\right\rangle$. Moreover the area function $A(t)=\int_{M} d v_{F_{t}^{\star} h}$ satisfies

$$
A^{\prime}(0)=-n \int_{M} H f d v
$$

We say that $F$ is preserving the volume if $V(t)=V(0)$ in a neighbourhood of 0 and in this case we have $\int_{M} f d v=0$. Conversely, for all smooth functions $f$ so that $\int_{M} f d v=0$, there exists a preserving volume variation so that $f=\left\langle\frac{\partial F}{\partial t}(0, x), \nu\right\rangle$. The following assertions are equivalent

(i) The immersion $\phi$ is a critical point of the area (i.e. $A^{\prime}(0)=0$ ) for all variations with preserving volume.

(ii) $\int_{M} H f d v=0$ for any smooth function so that $\int_{M} f d v=0$.

2010 Mathematics Subject Classification. 53C24, 53C42.

Key words and phrases. Stable constant mean curvature, Hypersurface, Geodesic sphere. 
(iii) There exists a constant $H_{0}$ so that $A^{\prime}(0)+n H_{0} V^{\prime}(0)=0$ for any variation.

(iv) $\phi$ is of constant mean curvature $H_{0}$.

An immersion with constant mean curvature $H_{0}$ will be said stable if $A^{\prime \prime}(0) \geqslant 0$ for all variations preserving the volume. Now we consider the function $\mathcal{J}(t)$ defined by

$$
\mathcal{J}(t)=A(t)+n H_{0} V(t)
$$

Then, $\mathcal{J}^{\prime \prime}(0)$ depends only on $f$ and we have

$$
\begin{aligned}
\mathcal{J}^{\prime \prime}(0) & =\int_{M}|d f|^{2} d v-\int_{M}\left(\operatorname{Ric}^{N}(\nu, \nu)+|B|^{2}\right) f^{2} d v \\
& =\int_{M} f \Delta f-\int_{M}\left(\operatorname{Ric}^{N}(\nu, \nu)+|B|^{2}\right) f^{2} d v \\
& =\int_{M} f J f d v
\end{aligned}
$$

where $\operatorname{Ric}^{N}$ is the Ricci curvature of $N$ with respect to the metric $\bar{g}$ and $J$ is the socalled Jacobi operator defined by $J f=\Delta f-\left(\operatorname{Ric}^{N}(\nu, \nu)+|B|^{2}\right) f$. It is well known that $\phi$ is a stable constant mean curvature immersion if and only if $\mathcal{J}^{\prime \prime}(0) \geqslant 0$ for any smooth function $f$ so that $\int_{M} f d v=0$ or equivalently if $J$ is a non-negative operator.

Barbosa and Do Carmo [1 proved that the only stable closed CMC hypersurfaces of the Euclidean space are the round spheres. This result was extended later by Barbosa, Do Carmo and Eschenburg [2] for spheres and hyperbolic spaces. In 4], Grosjean with the first author considered the stablity of CMC hypersurfaces in Riemannian manifolds with (non-constant) bounded sectional curvature. After proving a pinching result for the first eigenvalue of the Laplacian, they were able to show that a closed stable CMC hypersurface of a Riemannian manifold with bounded sectional curvature and contained in a geodesic ball of sufficiently small radius is close to a geodesic sphere. Namely, close means here diffeomorphic and almost-isometric to a geodesic sphere of appropriate radius (depending upon the mean curvature).

The aim of this short note is to show that the assumption of being stable can be relaxed to almost stable in the result of [4]. By almost stable, we mean that the Jacobi operator $J$ is not supposed to be non-negative but greater than some small negative constant

$$
\int_{M} f J f d v \geqslant-n \varepsilon \int_{M} h^{2} f^{2} d v
$$

for any smooth function $f$ so that $\int_{M} f d v=0$, where $h=\sqrt{\|H\|_{\infty}^{2}+\delta}$. Note that $h^{2}$ appears in the right hand side of the almost stablility condition for homogeneity reasons.

From now on, we assume that the sectional curvature of $(N, \bar{g})$ satisfies $\mu \leqslant \operatorname{Sect}_{N} \leqslant \delta$, for $\mu \leqslant \delta$ two real constants. Before stating the main result of this 
note, we introduce the following function

$$
s_{\delta}(r)= \begin{cases}\frac{1}{\sqrt{\delta}} \sin \sqrt{\delta} r & \text { if } \delta>0 \\ r & \text { if } \delta=0 \\ \frac{1}{\sqrt{|\delta|}} \sinh \sqrt{|\delta|} r & \text { if } \delta<0\end{cases}
$$

as well as the following set: $\mathcal{H}_{V}(n, N)$ is the space of all Riemannian compact, connected and oriented $n$-dimensional Riemannian manifolds without boundary isometrically immersed into $\left(N^{n+1}, \bar{g}\right)$ which satisfy the following hypothesis on the volume : $V(M) \leqslant \frac{c \omega_{n}}{\delta^{n / 2}}$ if $\delta>0$ and $V(M) \leqslant c \omega_{n} i(N)^{n}$ if $\delta \leqslant 0$ for some constant $c$. For more convenience we take $1 / \sqrt{\delta}=+\infty$ if $\delta \leqslant 0$. This condition on the volume is required to apply the result of [4] (see Theorem 2.1 below) about the pinching of the first eigenvalue of the Laplacian. This condition comes from the extrinsic Sobolev inequality of Hoffman and Spruck [6] which is used in the proof of that pinching result.

The main result of this note is the following:

Theorem 1.1. Let $\left(N^{n+1}, \bar{g}\right)$ be a $(n+1)$-dimensional Riemannian manifold whose

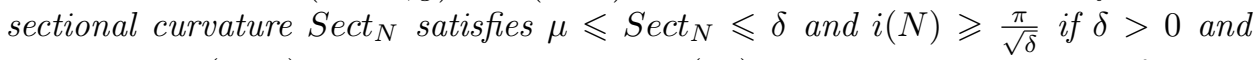
let $M \in \mathcal{H}_{V}(n, N)$. Let us assume that $\phi(M)$ lies in a convex ball of radius $\min \left(\frac{\pi}{8 \sqrt{\delta}}, \frac{i(N)}{2}\right)$. Let $\varepsilon<\frac{1}{12}, q>n$ and $A>0$. Let assume that $V(M)^{1 / n}\|B\|_{q} \leqslant$ $A$ for $\delta \geqslant 0 \quad$ (resp. $\max \left(\frac{H}{h}, V(M)^{1 / n}\|B\|_{q}\right) \leqslant A$ for $\left.\delta<0\right)$. Then there exist positive constants $\alpha:=\alpha(q, n), K:=K(n, q, A)$ and $R_{0}(\delta, \mu, \varepsilon)$ such that if $\phi$ is of constant mean curvature $H$ and almost stable in the sense of (1), $\varepsilon^{\alpha}<1 / K$ and $\phi(M)$ is contained in a convex ball of radius $R_{0}(\delta, \mu, \varepsilon)$ ) then $M$ is diffeomorphic and $K \varepsilon^{\alpha}$-quasi-isometric to $S\left(p, s_{\delta}^{-1}\left(\frac{1}{h}\right)\right)$, that is there exists a diffeomorphism $F$ from $M$ into $S\left(p, s_{\delta}^{-1}\left(\frac{1}{h}\right)\right)$ so that

$$
\left.|| d F_{x}(u)\right|^{2}-1 \mid \leqslant K \varepsilon^{\alpha}
$$

for any $x \in M, u \in T_{x} M$ and $|u|=1$.

\section{Proof of the Result}

Let $M \in \mathcal{H}_{V}(n, N)$ and denote by $\phi$ the isometric immersion of $(M, g)$ into $(N, h)$. Moreover, let us assume that $\phi$ has constant mean curvature and is almost stable in the sense of 11 for some positive $\varepsilon$. Let $f$ be an eigenfunction associated with the first eigenvalue $\lambda_{1}(M)$ of the Laplacian on $(M, g)$. Since $\int_{M} f d v=0$ then condition (1) gives

$$
\lambda_{1}(M) \int_{M} f^{2} d v-\int_{M}\left(\operatorname{Ric}^{N}(\nu, \nu)+n H^{2}+|\tau|^{2}\right) f^{2} d v \geqslant-\varepsilon n h^{2} \int_{M} f^{2} d v
$$


where $\tau$ is traceless part of the second fundamental form $B$. Since $\mu \leqslant S e c t^{N}$, we deduce that

$$
\begin{aligned}
\lambda_{1}(M) & \geqslant n\left(H^{2}+\mu-\varepsilon h^{2}\right) \\
& \geqslant n h^{2}+n\left(\mu-\delta-\varepsilon h^{2}\right) \\
& \geqslant n h^{2}\left(1+\frac{\mu-\delta-\varepsilon h^{2}}{h^{2}}\right)
\end{aligned}
$$

and so

$$
n h^{2} \leqslant \lambda_{1}(M)\left(1+\frac{1}{\frac{h^{2}}{\delta-\mu+\varepsilon h^{2}}-1}\right) .
$$

Now, we set $R_{1}(\delta, \mu, \varepsilon)=s_{\delta}^{-1}\left(\sqrt{\frac{\frac{1}{2}-\varepsilon}{(\delta-\mu)\left(\frac{1}{2 \varepsilon}+1\right)}}\right)$. We recall that the extrinsic radius of $M$ is defined as the radius of the smallest ball containing $\phi(M)$. We have the following well-known lower bound for the extrinsic radius $R(M)$ (see [3] for instance)

$$
s_{\delta}(R(M)) \geqslant \frac{1}{h}
$$

for compact hypersurfaces of a Riemannian manifold of sectional curvature bounded from above by $\delta$. Now, if we assume that $\phi(M)$ is contained in a ball of radius $R_{1}$, then since $s_{\delta}$ is an increasing funtion, we get

$$
h^{2} \geqslant \frac{1}{s_{\delta}^{2}(R(M))} \geqslant \frac{1}{s_{\delta}^{2}\left(R_{1}\right)}=\frac{(\delta-\mu)\left(\frac{1}{\varepsilon}+1\right)}{\frac{1}{2}-\varepsilon} .
$$

From this, we deduce easily that

$$
\frac{1}{\frac{h^{2}}{\delta-\mu+\varepsilon h^{2}}-1} \leqslant 2 \varepsilon
$$

and so

$$
n h^{2} \leqslant \lambda_{1}(M)(1+2 \varepsilon)
$$

that is, $\left(\Lambda_{2 \varepsilon}\right)$ holds, where we denote by $\left(\Lambda_{\eta}\right)$ the following pinching condition

$$
n h^{2} \leqslant \lambda_{1}(M)(1+\eta)
$$

associated with the following upper bound for the first eigenvalue of the Laplacian proved by Heintze [5]

$$
\lambda_{1}(M) \leqslant n h^{2} .
$$

Now, we recall the following theorem proved by the first author and Grosjean in [4].

Theorem 2.1 (4]). Let $\left(N^{n+1}, \bar{g}\right)$ be a $n+1$-dimensional Riemannian manifold whose sectional curvature Sect satisfies $\leqslant \leqslant \operatorname{Sect}_{N} \leqslant \delta$ and $i(N) \geqslant \frac{\pi}{\sqrt{\delta}}$ if $\delta>0$. Let $M \in \mathcal{H}_{V}(n, N)$. Let us assume that $\phi(M)$ lies in a convex ball of radius $\min \left(\frac{\pi}{8 \sqrt{\delta}}, \frac{i(N)}{2}\right)$. Let $p_{0}$ be the center of mass of $M$. Let $\eta<1 / 6$, $q>n$ and $A>0$. Let us assume that $\max \left(V(M)^{1 / n}\|H\|_{\infty}, V(M)^{1 / n}\|B\|_{q}\right) \leqslant A$ for $\delta \geqslant 0 \quad\left(\right.$ resp. $\max \left(V(M)^{1 / n}\|H\|_{\infty}, \frac{\|H\|_{\infty}}{h}, V(M)^{1 / n}\|B\|_{q}\right) \leqslant A$ for $\left.\delta<0\right)$. Then there exist positive constants $C:=C(n, q, A)$ and $\alpha:=\alpha(q, n)$ such that if 
$\left(\Lambda_{\eta}\right)$ holds, $\eta^{\alpha}<1 / C$ and $\phi(M)$ is contained in the ball $B\left(p_{0}, s_{\delta}^{-1}\left(\sqrt{\frac{\eta}{\delta-\mu}}\right)\right)$ then $M$ is diffeomorphic and $C \eta^{\alpha}$-quasi-isometric to $S\left(p, s_{\delta}^{-1}\left(\frac{1}{h}\right)\right)$.

Let $\varepsilon<\frac{1}{12}$ and $\eta=2 \varepsilon<\frac{1}{6}$. We set

$$
R_{0}(\delta, \mu, \varepsilon)=\min \left\{s_{\delta}^{-1}\left(\sqrt{\frac{2 \varepsilon}{\delta-\mu}}\right), R_{1}(\delta, \mu, \varepsilon)\right\}
$$

and $K(n, q, A)=2^{\alpha(n, q)} C(n, q, A)$, where $\alpha, C$ and $R$ are the constants given by Theorem 2.1 and $R_{1}$ is defined in the beginning of the proof.

If we assume that $\phi(M)$ is contained in a ball of radius $R_{0}(\delta, \mu, \varepsilon)$, by the definition of $R_{0}(\delta, \mu, \varepsilon)$, we have $R_{0}(\delta, \mu, \varepsilon) \leqslant R_{1}(\delta, \mu, \varepsilon)$ and so we get from the above computation that $\left(\Lambda_{2 \varepsilon}\right)$ holds. Moreover, we also get that $\phi(M)$ is contained in a ball of radius $s_{\delta}^{-1}\left(\sqrt{\frac{2 \varepsilon}{\delta-\mu}}\right)$. In addition, if we assume that $\varepsilon^{\alpha}<1 / K$, then, from the definition of $K$, we get that $(2 \varepsilon)^{\alpha}<1 / C$ and Theorem 2.1 (applied with $\eta=2 \varepsilon)$ gives that $M$ is diffeomorphic and $C \eta^{\alpha}$-quasi-isometric to $S\left(p, s_{\delta}^{-1}\left(\frac{1}{h}\right)\right.$. Since $C \eta^{\alpha}=K \varepsilon^{\alpha}$, by the definition of $K$, Theorem 1.1 is proven.

Remark 2.2. In the case of the Euclidean space, the result of Theorem 1.1 was proven in [7] and in this case, there is no assumption of being contained in a smal ball. Theorem 1.1 gives an analogue of the result of [7] for the hyperbolic space as well as the half-sphere. Indeed, if $\mu=\delta$, then $R_{0}(\delta, \mu, \varepsilon)=+\infty$ since both $s_{\delta}^{-1}\left(\sqrt{\frac{2 \varepsilon}{\delta-\mu}}\right)$ and $R_{1}(\delta, \mu, \varepsilon)$ are $+\infty$.

\section{Acknowledgements}

Second author gratefully thanks the support of National Post-doctoral Fellowship (File no. PDF $/ \mathbf{2 0 1 7 / 0 0 1 1 6 5 ) ~ f r o m ~ S c i e n c e ~ a n d ~ E n g i n e e r i n g ~ R e s e a r c h ~ B o a r d , ~}$ Government of India.

\section{REFERENCES}

[1] J.L. Barbosa \& M.P. Do CARmo Stability of Hypersurfaces with Constant Mean Curvature, Math. Z., 185, No. 3, (1984), 339-353.

[2] J.L. Barbosa, M.P. do Carmo \& J. Eschenburg, Stability of hypersurfaces of constant mean curvature in Riemannian manifolds, Math. Z. 197 (1) (1988), 123-138.

[3] C. Baikoussis \& T. Koufogiorgos, The diameter of an immersed Riemannian manifold with bounded mean curvature, J. Austral. Soc., (Series A), 31 (1981), 189-192.

[4] J.F. Grosjean \& J. Roth, Eigenvalue Pinching and Application to the Stability and the Almost Umbilicity of Hypersurfaces, Math. Z. 271(1) (2012), 469-488.

[5] E. Heintze, Extrinsic upper bound for $\lambda_{1}$, Math.Ann., 280, (1988), 389-402.

[6] D. Hoffman \& J. Spruck, Sobolev and isoperimetric inequalities for Riemannian submanifolds, Comm. Pure and Appl. Math., 27 (1974), 715-727.

[7] J. Roth \& J. Scheuer, Pinching of the first eigenvalue for second order operators on hypersurfaces of the Euclidean space, Ann. Glob. Anal. Geom. 51(3) (2017), 287-304. 
(J. ROTH) Laboratoire D'Analyse et de Mathématiques Appliquées, UPEM-UPEC, CNRS,

F-77454 MARNE-LA-VALLÉE

E-mail address: julien.roth@u-pem.fr

(A. UPADhyay) Indian Institute of Science, Department of Mathematics, Bangalore, 560012, INDIA

E-mail address: abhi.basti.ipu@gmail.com, abhitoshu@iisc.ac.in 